\title{
Dynamic Route Planning (DRP) Module for Distribution Business
}

\author{
Darshana T. Dayaratne ${ }^{\# 1}$, Sachini Gunasekara ${ }^{* 2}$ \\ ${ }^{\# 1}$ Department of Information Technology, CINEC Campus \\ Millenium Drive, IT Park, Malabe, Sri Lanka. \\ ${ }^{1}$ darti02@gmail.com \\ ${ }^{2}$ Sachini.Vindya@cinec.edu
}

\begin{abstract}
In the Sri Lankan logistics industry, delivery route planning is a very important and critical task. It requires many skilled labours and is time-consuming as well. The project aims to develop a Dynamic Route Planning (DRP) Algorithm to computerize the task and make it fast and efficient. The system comprises many features, including identifying the fastest route to deliver goods to all the given delivery locations (drop points). It also captures the cargo volume, vehicle capacity and provides an indication of the number of vehicles required to complete the full delivery by running a calculation using the volume of the cargo and truck capacity. The biggest challenge faced in developing the algorithm is calculating the distance among the delivery locations electronically. There are many electronic maps found online but each one has its own drawbacks. Most of the maps do not get an update very frequently, and changes on roads are not available. There are some maps, which are restricted to use in certain countries and not allowed to use worldwide. The algorithm and application what this project expecting to develop must be available to use in any country. Finally, we derived to a point where we can use Google maps which has minimal negative points. This project uses Google Maps as a tool when it is calculating the distances between two points, and inbuilt intelligence of Google Maps to identify the fastest route between two delivery points (drop points). At the beginning of the development of the algorithm, knowledge extracted by studying Dijkstra's Algorithm, which is used in Google Maps, supported to identify the direction of experiments. There are certain limitations in the use of Google Map APIs. As well as the number of hits made per day in the free version is limited and identifying different graded roads such as " $A$ " \& " $B$ " are some of them. Users can upload the details of the cargo and delivery location (drop point) details to the application. The application will then generate the fastest route in a report form or plotted in the map.
\end{abstract}

Keywords - Dynamic Route Planning, Cubic Meter, Application Programming Interface (API), Drop points

\section{INTRODUCTION}

The business who is doing B2B (Business to Business) or B2C (Business to Customer) are using third party logistics providers to provide services for their business. Warehousing, Cargo Distribution are some of the services that third party service providers can provide. These two services always go hand in hand. Because, warehousing is, storing cargo in a facility and dispatch those based on the orders. Transportation is, distributing the card in which they were dispatched from the warehouse to another businesses or customers.

Most of the distribution companies, maintain delivery schedules with static routes. When we analyze this distribution methodology and after talking to industry experts, we have identified, these scheduled deliveries can lead to customer dissatisfactions [1]. That can create delays in deliveries. Because, if the warehouse missed the scheduled delivery date when they dispatch the order, then the order will be delivered by the other week as a result the delivery is delayed by a week. This delay can be a week if the distribution company maintains a weekly schedule. If they are maintaining monthly schedules, that can lead to a one-month delay as well.

A solution is needed to be derived to do the deliveries in dynamic routes. That is without following scheduled deliveries, distributor to follow routes dynamically based on the orders for a particular day. In that way, the distribution company can minimize the delays up to a great extent. However, route planning will be the challenge because the number of combinations which can be available in arranging the delivery points (drop points), going up to millions depending on the number of them. It is not possible for humans to calculate all the combinations and find the fastest route.

As a solution, this research proposed an algorithm to find the fastest route to reach all the given delivery points. In this algorithm, users must be able to upload all the delivery points (drop points), and the algorithm will break the locations into multiple zones based on their geographical locations and then calculate the fastest routes within the zones. A prototype can prove the accuracy of the algorithm. For the prototype, it is given a higher priority for the algorithm and uses a very basic database and a programming language to develop it.

The rest of this paper is organized as follows: In section II, the literature review is discussed. Section III describes the proposed algorithm and implementation result, and section IV describes the conclusion.

\section{LITERATURE REVIEW}

The primary source of knowledge for this project is the specialised online application available in the market and specialised tools related to route planning.

While researching about available applications, two products were identified in the industry which allows anyone to download free demo versions and which has detailed documentation available about the product. Other applications need to pay and download the trial version or contact sales centre to get further details after registering the business at their website.

\section{TrustTrack}

TrustTrack is a Fleet Management Solution from Ruptela, headquarters located in Lithuania [2]. Ruptela was established in the year 2007 and founded by Andrius Rupsys. This is a company with a vision to be within the top 40 companies in transport telematics aftermarket globally by 2022 . The company has started with three members, and now it has grown to 220 total staff. Today, Ruptela 
develop, design and manufacture hardware, firmware and software products for fleet management and GPS tracking. Ruptela provides ready-to-use telematics solutions. Ruptela is an ISO 9001:2015 certified company, and ISO 9001:2015 is internationally recognised as the world's leading quality management standard.

TrustTrack consists of six main features.

Vehicle Tracking: Through GPS technology, the system can track the vehicle location, trip history, and routes vehicle travelled, send messages \& tasks to the driver and send notifications.

Connect \& Integrate: Users can retrieve details of drivers, vehicles, tasks, routes, KPIs and all other data and display in a single platform. TrustTrack can integrate with other third-party software and web services using APIs.

Telematics: Retrieve data from digital tachograph, CAN bus, monitor fuel level and consumption, rate drivers by their driving style.

User Friendly: TrustTrack is a user-oriented web-based platform with a simple and native user interface. It is a fast and proactive platform.

Mobile Apps: TrustTrack mobile app is very useful option in this solution. Users can log in to the mobile app as the fleet management or as the driver. The features what app returns changes based on the login method.

Admin Panel: Admin panel is a user management platform for tracking service providers. It makes it easier to create, edit, control and bill users.

In addition to those mentioned above six main features, users can utilize few other facilities as mentioned below.

- Check the status of the fleet.

- Remotely download data from the tachograph

- Manage drivers, working time, idle time \& resting time.

- Monitor detailed fuel levels and consumptions.

- Rank drivers driving style wasted fuel and efficiency.

- Create the tasks and routes for the particular job and send it to the driver via the system.

- $\quad$ Receive an alert when the vehicle needs to be serviced.

- Manage the vehicle within the pre-set working area using geofencing/zoning technology.

- Communicate with drivers through voice or text messages or send/receive files through the application.

- Useful dashboard

- Customized reports.

TrustTrack mainly focuses on tracking the fleet and managing the vehicle fleet. The challenge that the industry currently facing is identifying the most suitable route to reach the locations from the distribution location. There are many applications bundled with regular GPS solutions to track the vehicle location, check the fuel levels, monitor the route that the vehicle is using, etc. TrustTrack is a reactive solution and industry is looking for a proactive solution. Therefore, the TrustTrack application does not fulfil the requirement that industry is looking for [2].

\section{Locus}

Locus is an Indian company who is developing IT solutions for the logistics industry. The company started in 2014 by developing an application for a personal requirement. This application was for the women in India for their safety. Then this requirement came for the food delivery companies to monitor their deliveries, and it gave a huge opening for a new avenue in the logistics industry. Locus mainly focus on automation solutions for the logistic industry.

Locus on the Road module is designed with some features as mentioned below.

\section{- Detailed and Easy to Use User Interface}

Hassel free tasks navigation, with customer details including accurate geocoded locations.

- $\quad$ Electronic Proof Of Delivery

Capture customer signature, product image or QR code at the point of delivery to confirm the goods delivery.

- Reschedule and Cancel Orders

Easily reschedule or cancel deliveries, the basis of customer availability within the app.

- Partial Pickups and Deliveries

The distributor can cancel part of the order to enhance customer experience and reduce logistics costs.

This application has been extended to a mobile app where the drivers can access it from any location [3].

Since the Locus application is mainly focused on the accuracy of goods delivery and traceability, it is not giving intelligence to find the fastest route to reach multiple locations. This tool can be used to stop the use of paper documents and transferred them to a digital platform.

\section{Methodology}

After identifying the industry requirements and analysing the existing applications, it is decided that developing an algorithm to plan routes dynamically is the only solution to improve the efficiency in cargo distribution. Google Maps are the most common and frequently updating application available online [4]. To get the support of Google Maps, it is necessary to learn about the Google Developer Console.

\section{Google developer console}

Google developer console, previously called as google code, is google's site for software development tools, APIs and technical resources. Additionally, this site contains documentation on using Google APIs which developers can refer and learn how to use the APIs. Sample codes are helpful to proceed with trials. Blogs \& group discussions are helpful to understand the possible errors, their solutions and share knowledge.

Google developer consoler offers APIs for almost all the google's popular consumers such as google maps, YouTube and google apps. This site features a variety of developer products and tools especially for developers.

\section{Google distance matrix API}

The distance matrix API is a service that is provided by Google to get the travel distance and time for a matrix of origins and destinations. Since the security is an important fact, applications can use https and prevent from snooping or tampering. The respond of the request can be received in json or xml files. If the developer mention "json" as the output format in the request parameter, respond receive as json, if it mentioned as $\mathrm{xml}$, respond receives in $\mathrm{xml}$.

\section{Request syntax}

https://maps.googleapis.com/maps/api/distancematrix/json?origins = $<$ origin latitude $>$, $<$ origin longitude $>\&$ destinations $=<$ destination latitude $>, \quad<$ destination longitude $>\quad$ \&language $=$ en-en \& sensor=false $\&$ avoid=highways $\&$ key $=<$ key $>$

\section{Parameters}

Origin : this is a mandatory parameter in google distance matrix API. When the application wants to check the distance between two geographical locations, the starting location is the origin. When the application passes the parameter of the origin, application can pass either the location address or the latitude and longitude co-ordinations. It is recommended to use the latitude and longitude co-ordination rather using the location address because in a location 
address, there is no specific number of elements, and each element needs to be separate by the plus "+" sign. Furthermore, if the address is not registered in google, API cannot identify the correct geographical location and return the correct distance.

Destination: this is a mandatory parameter of google distance matrix API. When the application wants to check the distance between two geographical locations, the ending location is the destination. Similarly, to parameter origin, this parameter also can be passed by the location address or latitude \& longitude co-ordination. Again, it is recommended to use the latitude \& longitude coordination. In this parameter, the application can pass multiple locations and then API can return the total distance to travel all the locations starting from the given origin.

Key : this is the last mandatory parameter that google distance matrix requires. To access google APIs, the application should communicate through a registered account. Users need to create the google account and enable the APIs. Google issues a key once the registration is done and google use that as an authentication while application is accessing the API. Additionally, Google maintains a quota for each registration and that quota also managed by the key.

There are a few more optional parameters which users can use in their applications.

Mode: this is the parameter which application inform the API the mode of travel. The default is "driving". Additional to "driving" users can use "walking", "bicycling" \& "transit".

Language: google API can respond in multiple languages. Default is English. (refer the appendix 1 for other languages)

Avoid: while google is finding the path between two locations, there can be some options to be avoided. Such as roads with tolls, expressways, etc. (refer the appendix ii for all the possible avoids)

Units: This parameter sets the type of measurement to return the distance in API response. Google API supports two types of units that are metric \& imperial.

There are few more optional parameters that can be used with google API such as traffic-mode, transit-mode, transit-routingpreference, etc. [5]

\section{1) Identifying the Requirement}

In Sri Lanka, logistics is a newly booming industry. Cargo distribution is one of the major tasks in logistics. Cargo distribution in modern trade or general trade is a daily requirement. There are thousands of deliveries happening in Sri Lanka every day, and all these distributions need a perfect route planning [1] [6].

Currently, all the route planning happening through standard delivery schedules, and there are hidden costs and wastages within the distribution. Skilled route planners are an essential requirement in manual route planning, and it is an unavoidable cost for a company [1][6]. Humans can make mistakes, and that can lead to a loss for the company as well.

Dealing with the customers always need proof to ensure the route that the company used is the most efficient route for their cargo distribution. System generates route plans always gives an additional confidence for the customers, and it can be an added advantage to the company to get many more businesses and compete with the competitors within the industry [1] [6].

By the research conducted for this project, it was clear that dynamic route planning always needs to go with online maps that has the accuracy of $99 \%$ [1][6].

\section{2) Limitation}

There are a few identified limitations in this project.

Geo-coordinates - This algorithm totally depends on the distances between drop points. To calculate the drop points, it is compulsory to have the geo-coordinates of each and every drop point. The system cannot continue without the geo-coordinates. There are Google APIs that returns the geo-coordinates of the location if the system can pass the postal address to Google as a parameter. However, google can return wrong coordinates or may not return the coordinates if the postal address is not registered/ found on google maps. In this phase, this project does not facilitate to do the route planning based on the drop point postal address.

There is a limitation in the number of hits that Google maps can allow per day in its free version. If the user plans to have many route planning sessions with many drop points on a daily basis, the user needs to purchase the google API license. Some routes google picks are not accessible by bigger trucks. There is no parameter in google API to avoid that type of route. Therefore, there are situations where truck/lorry cannot travel in the route that google suggest and, in that instance, the route plan that this algorithm gives may have a slight difference from the actual route that the truck/lorry can travel.

Google always returns the distance of the fastest route and not the shortest route. The fastest route depends on the traffic situation at the time of planning. Users can do the planning, may be nighttime or the previous day of the actual delivery. However, the distribution can happen at a different time. The traffic situation at the time of planning and actual delivery can be different. Therefore again, the planned route can differ from the actual route.

\section{3) Algorithm}

\section{Problem}

There are a set of delivery locations that are changing randomly. Need to find the fastest route to reach all the location from the distribution point.

\section{Assumptions}

There are a few assumptions in this algorithm.

1. User can upload the correct geo co-ordinates of all the locations, and all the locations are valid location and application do not verify it.

2. All the geographical locations are accessible by road. No other transport mediums (sea and air) to be used.

3. Application do not need to warn if the user uploads a location which needs another transport medium to reach the location.

4. Only single-origin location available for a route plan and no multiple pick-up points.

5. Application does not verify the number of pick-up points.

6. User has updated all the available vehicle types in the Fleet Master Table

\section{4) Tools}

To find the fastest route, need a solution to find the distances between the locations, which are changing randomly. Electronic Maps (online maps) can be used as a tool to find the distances between the locations. When a third-party program communicates with the online maps, it is necessary to pass the Longitude \& Latitude coordinates of the locations or location's addresses as parameters. Then the map can return the distance between the two locations. Here Google Maps APIs can be used to communicate with Google Maps, through a third-part program and read the response. Google APIs can return the respond through json or xml Communicating using json or $\mathrm{xml}$, third-party program can identify the distances, avoids or include/exclude roads with tolls, etc.

Since the purpose of the project is to test the algorithm, the prototype can be developed using any programming language. In this project, Visual Basic 6 has been selected as the programming language for the development of prototype and Crystal Report as the reporting tool. 


\section{5) Process}

Google Maps are already using an algorithm to identify the route between two geographical locations, and it is commonly used in the world. The studies of google maps and the algorithm used in that which is "Dijkstra's Algorithm" has been supportive to find the algorithm for "Dynamic Route Planning". Since the abovementioned algorithm is used to find the shortest path between two locations. Started with applying the Dijkstra's Algorithm to Dynamic Route Planning as it is. Then the result was, the system is returning the best single route to serve all the delivery locations. However serving all the delivery locations using a single route is not practical, and it is not benefitting to the cargo distributor. Then started to find a solution to break the route into zones using the proposed method. Finally, the method shows in fig. 1 returned the best route to serve any given locations.

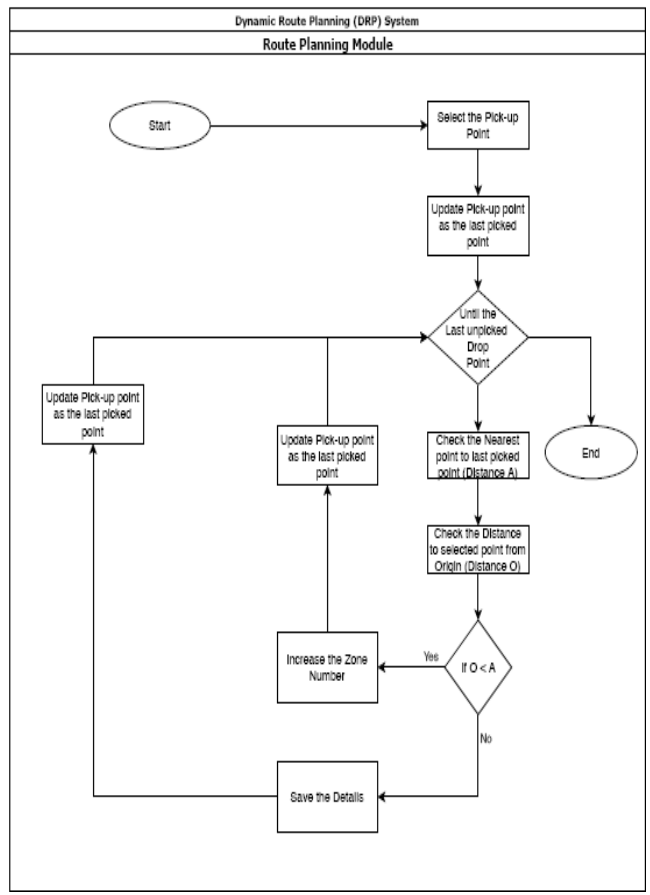

Fig. 1- Dynamic Route Planning Process

\section{6) System Testing}

Now the project has come to the most important stage where the prototype is developing and testing the final results. After the problem is identified, done the successful research on existing solutions and identified the advantages and disadvantages of those solutions. Literature reviews allowed to get a good knowledge about the existing tools and logics what those tools are using when they calculate the fastest distances to a location from another location. By improving those methodologies, this project was able to come up with a perfect algorithm to identify the fastest route to reach multiple locations from a single distribution location. This is the first time this type of the logic is implementing in Sri Lanka and testing multiple scenarios can confirm the accuracy of the algorithm.

Test Case 1

They are distributing from Warehouse in Muthurajawela Industrial Zone to 17 locations. The distributing locations are as follows in Table I.
TABLE I

LIST OF DROP LOCATIONS

\begin{tabular}{|l|r|r|}
\hline \multicolumn{1}{|c|}{ Location Name } & \multicolumn{1}{c|}{ Latitude } & \multicolumn{1}{c|}{ Longitude } \\
\hline S051-Ahangama & 5.97331905 & 80.36201477 \\
\hline S053-Alawwa & 7.29728699 & 80.23690796 \\
\hline S017-Aluthgama & 6.43073988 & 79.998909 \\
\hline S054-Ambalangoda & 6.23655796 & 80.05323029 \\
\hline S055-Ambalanthota & 6.12268496 & 81.02935791 \\
\hline S122-Balangoda 2 & 6.6476123 & 80.7005585 \\
\hline S025-Bandarawela & 6.83047819 & 80.98975372 \\
\hline S026-Badulla & 6.99085188 & 81.05352783 \\
\hline S150-Galle1 & 6.05007696 & 80.22026062 \\
\hline S323-Hambanthota & 6.12510395 & 81.12612152 \\
\hline S329-Hikkaduwa & 6.14270306 & 80.0996933 \\
\hline S381-Jaffna & 9.66859722 & 80.01738739 \\
\hline S456-Kalpitiya & 8.2323128 & 79.7609274 \\
\hline S006-Kaluthara & 6.58225012 & 79.9598465 \\
\hline S419-Kataragama & 6.4150548 & 81.33216858 \\
\hline S514-Mannar & 8.9767116 & 79.9142487 \\
\hline S658-Polgahawela & 7.33611917 & 80.30046082 \\
\hline
\end{tabular}

The system should group these 17 locations into zones as, mentioned in Point 5 - Process. The system processed the above uploaded locations as shown in figure 2, and the algorithm has grouped those 17 locations into 4 zones.

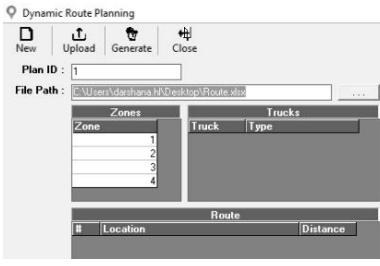

Fig. 2- Routing Zones

Zone 1

The system has identified two delivery points in Zone 1 from Spectra Distribution

1. S053:Alawwa

2. S658:Polgahawela

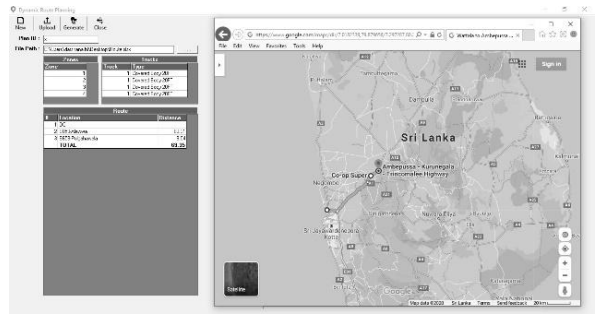

Fig. 3- Zone 1 Route 
Zone 2

System has identified 9 delivery points in Zone 2

1. S006:Kaluthara

2. S017:Aluthgama

3. S054:Ambalangoda

4. S329:Hikkaduwa

5. S150:Galle -1

6. S051:Ahangama

7. S055:Ambalanthota

8. S323:Hambanthota

9. S419:Katharagama

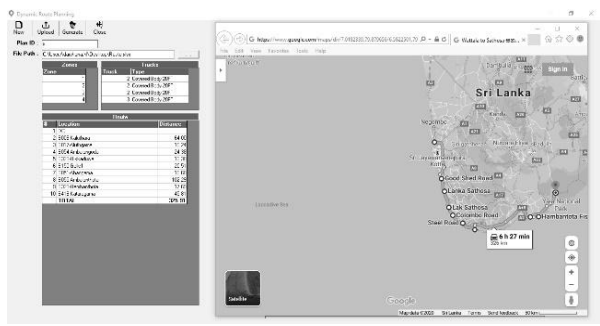

Fig. 4-Zone 2 Route

Zone 3

System has identified 3 delivery points in Zone 3

1. S122:Balangoda 2

2. S025:Bandarawela

3. S026:Badulla

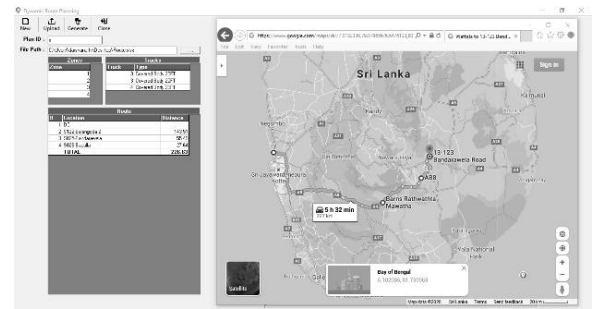

Fig. 4-Zone 3 Route

\section{Zone 4}

System has identified 3 delivery points in Zone 4

1. S456:Kalpitiya

2. S514:Mannar

3. S381:Jaffna

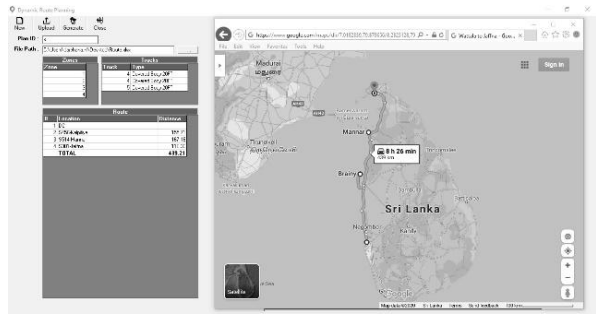

Fig. 5- Zone 4 Route

\section{CONCLUSIONS}

The Dynamic route-planning algorithm was developed with the learning of Dijkstra's Algorithm. Dynamic route planning algorithm is an extension of Dijkstra's algorithm. This algorithm helps users to plan their daily deliveries in an easy and most efficient way within a small period. Implementing this algorithm with an online web-based interface, it can use as a common route planning online platform for any distribution centre. Furthermore, the density data were stored in database and, analysed to understand volume of orders being placed in geographical areas within the country and forecast about the demand and more information.

System allows users to add/remove orders at any time and re-plan the routes within a small-time frame. This increases the flexibility of the delivery and it is a value-added service for any distribution company. When the vehicle fleet follows efficient route in distribution, the fuel cost and time can be reduced which can bring much more benefits to the business. On-time deliveries will positively impact on the company brand and increase the trust and reliability of the company from customer point of view.

\section{ACKNOWLEDGMENT}

I would like to express my sincere gratitude to everyone who helped me throughout the course with guidance and providing knowledge to complete my project.

I would like to take this opportunity to thank Mr. Fawmy Siddhik, general manager at Spectra Logistics, who supported me with gaining knowledge in Supply Chain Management Systems and Cargo Distribution. His experience and knowledge helped me to identify the problems in route planning and the importance of systemizing it. Despite from his fully packed daily work routine, he always managed to provide his feedback on my observations and back me up whenever I am down.

\section{REFERENCES}

1 F. Vyacheslav, "Amconsoft," Amconsoft, 0903 2020. [Online]. Available: https://amconsoft.com/real-time-dynamic-routeoptimization-for-delivery-business/. [Accessed 0204 2020].

2 Ruptela, "Ruptela Transport Telematic," 2019. [Online]. Available: https://www.ruptela.com/transport-managementsystem/. [Accessed 2201 2020].

3 Locus, "Locus," 2019. [Online]. Available: https://locus.sh/. [Accessed 0602 2020].

4 K. K. BECKIUS, "TripSavvy," TripSavvy, 0603 2019. [Online] Available: https://www.tripsavvy.com/free-driving-directionsonline-1601072. [Accessed 2601 2020].

5 Google, "Google - Developer Guide," 2020. [Online]. Available: https://developers.google.com/maps/documentation/distancematrix/intro. [Accessed 1502 2020].

6F. Siddhik, Interviewee, Business Development Manager - Spectra Logistics. [Interview]. 15022020.

7W. KENTON, "Investopedia," 2020. [Online]. Available: https://www.investopedia.com/terms/r/randd.asp. [Accessed 1206 2020].

8W. KENTON, "Investopedia," 2019. [Online]. Available: https://www.investopedia.com/terms/i/inventory.asp. [Accessed 03 02 2020]. 
9 Villanova University, "Villanova University," 2019. [Online]. Available: https://www.villanovau.com/resources/businessanalysis/business-analyst-job-description/. [Accessed 1306 2020].

$1 \mathrm{~V} . \quad J o s h i, \quad$ "Bedium," 2017. [Online]. Available: https://medium.com/basecs/finding-the-shortest-path-with-a-littlehelp-from-dijkstra-613149fbdc8e. [Accessed 2812 2019].

$1 \mathrm{U}$. Eriksson, "ReQtest," 2012. [Online]. Available: https://reqtest.com/requirements-blog/functional-vs-nonfunctional-requirements/. [Accessed 1506 2020].

1 TechTerms, "TechTerms," 2014. [Online]. Available: https://techterms.com/definition/faq. [Accessed 1506 2020].

1 S. S, "keydifferences," 203 2016. [Online]. Available: https://keydifferences.com/difference-between-code-of-ethics-andcode-of-conduct.html.

1S. Das, "SupplyChain 24 7," 2019. [Online]. Available: https://www.supplychain247.com/article/8_factors_to_consider_w hen_choosing_route_optimization_software. [Accessed 1801 2020].

1 S. F. \&. R. Steiner, "Science Direct," 2015. [Online]. Available: https://www.sciencedirect.com/topics/computer-science/casedescription. [Accessed 1006 2020].

1 R. Pichler, "Project Management.com," 2013. [Online]. Available: https://www.projectmanagement.com/articles/278628/Scenariosand-Storyboards. [Accessed 1006 2020].

1 i. O.-R. I. V. Rahul Kala, "ScanDirect," 2016. [Online]. Available: https://www.sciencedirect.com/topics/engineering/route-planning. [Accessed 1903 2020].

1 P. Duquenoy, "Finnish Information Processing Association," 912 2014. [Online]. Available: https:/tivia.fi/wpcontent/uploads/2019/05/TIVIA-ethics-code-unofficial-

translation.pdf.

1 Nishadha, "Creately," 2019. [Online]. Available: https://creately.com/blog/diagrams/use-case-diagram-tutorial/.

[Accessed 1206 2020].

2 N. Geographic, "National Geographic," 2015. [Online]. Available: https://www.nationalgeographic.com/animals/fish/group/freshwate r-fish/. [Accessed 15112020 ].

2 Monika, "BOYO," 2020. [Online]. Available: https://blogonyourown.com/what-is-blog/. [Accessed 1506 2020].

2 M. MURRAY, "The Balance Small Business," 2018. [Online]. Available: https://www.thebalancesmb.com/route-planning2221322. [Accessed 0501 2020].

2 L. Kramer, "BizFluent," 1907 2019. [Online]. Available: https://bizfluent.com/facts-6116651-code-ethics-important.html.

2 K. Leonard, "Chron," 1202 2019. [Online]. Available: https://smallbusiness.chron.com/importance-creating-code-ethicsbusiness-3094.html.

2 J. H. Hirsh, "Centre for International Governance Innovation," 09
07
2019.
[Online].
Available:

https://www.cigionline.org/articles/peril-and-potentialgdpr?gclid=EAIaIQobChMIue-Z7tyq5gIVkB0rCh0zEQ2EAAYASAAEgJ3MPD_BwE.

2 IESL, "Institute of Engineers Sri Lanka," 1612 2019. [Online]. Available:

https://iesl.lk/index.php?option=com_content\&view=article\&id=2 $0 \&$ Itemid $=138 \&$ lang=en.

2E. Cimolini, "routific," 2019. [Online]. Available: https://blog.routific.com/what-is-route-optimization. [Accessed 08 02 2020].

2 K.-T. (. C. Chung-Yang (Ric) Huang, "Science Direct," 2009. [Online].

Available: https://www.sciencedirect.com/topics/computer-science/dijkstraalgorithms. [Accessed 0501 2020].

2 A. Kim, "Medium," 2018. [Online]. Available: https://medium.com/@annkim_93227/what-is-human-computerinteraction-hci-3020e5c29e5b. [Accessed 1006 2020].

A. HAYES, "Investopedia," 2019. [Online]. Available: $3 \mathrm{https}: / / \mathrm{www}$. investopedia.com/terms/s/scm.asp. [Accessed 1412 ] 2019]. 